\title{
CXCR2/CXCR2 Ligand Biological Axis Impairs Alveologenesis During dsRNA-Induced Lung Inflammation in Mice
}

\author{
VEDANG A. LONDHE, JOHN A. BELPERIO, MICHAEL P. KEANE, MARIE D. BURDICK, \\ YING YING XUE, AND ROBERT M. STRIETER \\ Department of Pediatrics [V.A.L., R.M.S.], Division of Pulmonary and Critical Care Medicine [J.A.B., \\ M.P.K., M.D.B., Y.Y.X., R.M.S.], Department of Pathology and Laboratory Medicine [R.M.S.], Department \\ of Medicine [J.A.B., M.P.K., M.D.B., Y.Y.X., R.M.S.], David Geffen School of Medicine at UCLA, \\ Los Angeles, CA 90095
}

\begin{abstract}
The histologic phenotype of bronchopulmonary dysplasia (BPD) is characterized by decreased alveolization and is preceded by infiltration of activated neutrophils into the lung that can lead to sustained lung injury and potential interruption of normal lung development. Potential pathogens triggering early neutrophil influx include either prenatal or postnatal exposure to bacteria or viruses. Specific mechanisms recruiting neutrophils to the lung and subsequently decreasing alveolization during virusinduced lung inflammation and injury have not been fully elucidated. Because CXC chemokines, such as CXCL1 and CXCL2/3 acting through their putative receptor, CXCR2, are potent neutrophil chemoattractants, the authors investigated their role in dsRNA-induced lung injury and decreased alveolization, in which dsRNA (poly IC) is a well-described synthetic agent mimicking acute viral infection. Intratracheal dsRNA led to significant increases in neutrophil infiltration and lung injury at 72 hours and to decreased alveolization at 5 days after dsRNA exposure in newborn (10 days old) BALB/c mice, when com-
\end{abstract}

\section{ABSTRACT}

pared with controls treated and not treated with ssRNA (poly C). Expression of CXCL1 and CXCR2 paralleled neutrophil recruitment to the lung and preceded the decrease in alveolization. Inhibition of CXCR2/CXCR2 ligand interaction by pretreating dsRNA-exposed mice with an anti-CXCR2 neutralizing antibody significantly attenuated neutrophil sequestration and lung injury, and preserved normal alveolization. These findings demonstrate that the CXCR2/CXCR2 ligand biologic axis plays an important role during the pathogenesis of dsRNA-induced lung injury and decreased alveolization and may be relevant to the pathogenesis of BPD. (Pediatr Res 58: 919-926, 2005)

$\quad$ Abbreviations
BPD, bronchopulmonary dysplasia
dsRNA, double-stranded RNA
SSRNA, single-stranded RNA
ELR, glutamic acid-leucine-arginine
BAL, bronchoalveolar lavage

Decreased alveolization is a hallmark of bronchopulmonary dysplasia (BPD) and is usually preceded by an inciting inflammatory event early during the course of lung disease in preterm newborns $(1,2)$. Since its histopathologic description by Northway in 1967 as a fibrotic sequelae after mechanical ventilation, BPD has evolved into "new BPD" with minimal to no fibrosis but marked chronic alveolar hypoplasia $(3,4)$. Despite the evolution of this disease and significant advances in perinatal

Received November 22, 2004; accepted March 30, 2005.

Correspondence: Robert M. Strieter, M.D., Department of Medicine, Division of Pulmonary and Critical Care Medicine, and Department of Pathology and Laboratory Medicine, David Geffen School of Medicine at UCLA, Room 14-154 Warren Hall, Box 711922, 900 Veteran Ave, Los Angeles, CA 90095-1786; e-mail: rstrieter@mednet. ucla.edu.

This work was supported in part by the National Institutes of Health (NIH), grants HL66027 and P50HL67665 (R.S.), National Research Service Award (NRSA) Institutional Training Grant, T32 HD07549 (V.L.), and the NIH-sponsored UCLA Child Health Research Career Development Award (CHRCDA) (V.L.).

DOI: 10.1203/01.PDR.0000181377.78061.3E and neonatal care, the incidence of bronchopulmonary dysplasia (BPD) remains unchanged at approximately $30 \%$ in premature babies with very low birth weight (VLBW) $(<1500 \mathrm{~g})$ (5). Although efforts at preventing preterm delivery, introduction of antenatal steroids, surfactant therapy, and judicious use of oxygen and ventilator therapy all have improved survival of premature newborns; BPD continues to account for major morbidity and mortality in VLBW neonates.

The underlying inflammation preceding the development of BPD is thought to be a cumulative effect of hyperoxia and ventilator-induced lung injury in addition to prenatal or postnatal exposure to infectious agents. Potential pathogens that may trigger inflammation include bacteria (e.g., Ureaplasma urealyticum $(6,7))$ or viruses $(8-12)$. The mechanism of how inflammation leads to arrest of alveolar development has not been elucidated.

To date, whereas bacterial causes have been extensively studied, there is a dearth of information investigating the 
potential of viral pathogens leading to BPD. Tracheal aspirates of preterm infants have demonstrated a causal relationship between detection of CMV or adenoviral infection and subsequent development of BPD (9-11). A common feature of these viral infections is that they generate a cascade of pro- and anti-inflammatory cytokines that persist and contribute to chronic histopathologic changes within the lungs. Inflammatory changes due to viral infection are thought to result from the host immune response rather than secondary to viral replication $(13,14)$. Viral infections of epithelial cells are characterized by the generation of the pro-inflammatory molecule double-stranded RNA (dsRNA) during intracellular replication of viruses. dsRNA has been shown to induce activation of the neutrophil chemoattractant, IL-8 (IL-8/CXCL8), and RANTES (regulated on activation, normal $\mathrm{T}$ cells expressed and secreted) (15). In human subjects in vivo, elevated tracheal CXCL8 levels and neutrophil accumulation are found in airways of infants in whom BPD develops $(16,17)$. The potential role of dsRNA and induction of CXC chemokines during the development of BPD is unknown.

Murine KC/CXCL1 and MIP-2/CXCL2/3 are glutamic acidleucine-arginine-positive (ELR-positive) CXC chemokines; structural homologs of human GRO- $\alpha /$ CXCL1 and GRO- $\beta / \gamma /$ CXCL2/3, respectively; and are functional homologs of human ELR-postive CXC chemokines, such as CXCL8, GRO- $\alpha / \beta / \gamma /$ CXCL1/2/3, ENA-78/CXCL5, and GCP-2/CXCL6 (18). Both murine chemokines share the ability to signal through the CXC chemokine seven transmembrane $\mathrm{G}$ protein-coupled receptor, CXCR2 (18). Their human structural and functional homologs have been associated with early BPD $(16,19)$.

The purpose of the current study was to investigate the potential role of CXCR2 and its ligands in interrupting normal alveolization during dsRNA-induced lung inflammation and injury in a newborn mouse model. Newborn mice serve as an attractive animal model for studying alveolization because mice aged 0 to 21 days continue to have lungs that are progressing from a saccular to alveolar stage of development, the equivalent of human preterm infant lungs starting at 24 to 26 weeks' gestation (20). We hypothesized that the inflammation and subsequent arrest of alveolar development from intratracheal dsRNA treatment is caused, in part, by the expression of ELR-positive CXC chemokines through their interaction with their major receptor, CXCR2. To test this hypothesis, we injected intratracheal dsRNA into 10-day-old BALB/c mice to ascertain whether total neutrophil and chemokine responses of resultant lung injury resulted in decreased alveolization. We found that neutrophil recruitment, lung injury, and decreased alveolization caused by dsRNA exposure is dependent on the interaction between the ELR-positive CXC chemokine, CXCL1, and its receptor, CXCR2. We then blocked the effect of dsRNA exposure by pretreating animals with antibodies to specifically neutralize CXCR2 and neutrophil recruitment in a CXC chemokine-dependent manner and thereby decreased lung inflammation, decreased injury, and preserved normal alveolar development. Our animal model demonstrates the role of CXCR2/CXCR2 ligand interaction in dsRNA-induced lung inflammation and injury leading to decreased alveolization, a phenotype that resembles BPD.

\section{METHODS}

Reagents. For RNA instillation, dsRNA (Poly IC) and single-stranded RNA (ssRNA) (Poly C) were purchased from Sigma Chemical Co. Aldrich Corp. (St. Louis, MO) and reconstituted in sterile normal saline $(20 \mu \mathrm{g} / \mu \mathrm{L})$ and stored at $4^{\circ} \mathrm{C}$ before use.

For enzyme-linked immunosorbent assay (ELISA) experiments, biotinylated and nonbiotinylated anti-murine KC/CXCL1 and murine MIP-2/ CXCL2/3 were purchased from R\&D Systems (Minneapolis, MN).

In neutralization studies, polyclonal goat anti-murine CXCR2 was produced by the immunization of a goat with a peptide containing the ligand-binding sequence Met-Gly-Glu-Phe-Lys-Val-Asp-Lys-Phe-Asn-Ile-Glu-Asp-Phe-PheSer-Gly of CXCR2 (21). The goat was immunized with CXCR2 in multiple intradermal sites with complete Freund's adjuvant (CFA) followed by at least 3 boosts of CXCR2 in incomplete Freund's adjuvant (IFA) as previously described (21). Direct ELISA was used to evaluate antisera titers, and sera was used for Western blot, ELISA, and neutralization assays when titers had reached more than $1 / 1,000,000$. The CXCR2 protein sequence has been shown to contain the ligand-binding portion of the CXCR2 receptor (21). The anti-CXCR2 antibodies have been used previously to block mouse CXCR2 in vivo, and has been shown to detect CXCR2 by Western blot and fluorescenceactivated cell sorting analysis of neutrophils in vivo (21). The anti-CXCR2 antibody has been shown to be neutralizing using both in vitro neutrophil chemotaxis assay and in vivo by abrogating the influx of neutrophils into the peritoneum of normal mice in response to exogenous ELR positive murine CXC chemokines (21). In vivo administration of anti-CXCR2 antibodies inhibited pulmonary neutrophil sequestration in murine models of Aspergillosis, Nocardia, and Pseudomonas pneumonia and prevented the influx of neutrophils in urine and the kidney in a murine model of Escherichia coli urinary tract infection (21). Moreover, intraperitoneal administration of this antibody did not alter peripheral blood neutrophil counts (21). Previous studies comparing the anti-CXCR2 antibody to knockout animals for CXCR2 $\left(\mathrm{CXCR}^{-{ }_{-}}\right)$showed equal efficacy in attenuating pulmonary neutrophil sequestration $(22,23)$.

Murine model of dsRNA-induced lung injury. We used 10-day-old BALB/c mice to intratracheally inject either ssRNA or dsRNA using a modification of a previously described method $(24,25)$. Mice were anesthetized with ketamine $(60 \mathrm{mg} / \mathrm{kg})$ intraperitoneally; then, under sterile conditions, the anterior neck soft tissue was dissected to expose the trachea and $25 \mu \mathrm{L}$ RNA $(20 \mu \mathrm{g} / \mu \mathrm{L} ; 50 \mu \mathrm{g} / \mathrm{g}$ mouse weight) was injected via 26-gauge tuberculin needle and syringe attached to a Stepper® microinjector (Indicon, Inc., Brookfield, CT) into the trachea under direct visualization. Immediately after the instillation, the skin was apposed and closed using tissue adhesive and the mouse pups were allowed to recover from anesthesia before replacement into the cage with their mothers.

In separate experiments, animals received either $0.5 \mathrm{~mL}$ of anti-murine CXCR2 anti-sera, or $0.5 \mathrm{~mL}$ of normal goat serum control antibody intraperitoneally 24 hours before intratracheal injection and daily until time of killing as previously described (26). This study has been approved by the Institutional Animal Care and Use Committee (Chancellor's Animal Research Committee) of UCLA.

Lung bronchoalveolar lavage and tissue preparation. At time of sacrifice, 72 hours after intratracheal dsRNA or ssRNA treatment, mice were euthanized using intraperitoneal Pentobarbital $(100 \mathrm{mg} / \mathrm{kg})$ and a heparinized sample of blood was harvested. Age-matched 13-day-old untreated control mice were also similarly sacrificed for comparison. The thoracic cavity was then exposed and lungs were perfused free of blood with $1 \mathrm{~mL} 0.9 \%$ normal saline via the spontaneously beating right ventricle under constant pressure of $25 \mathrm{~cm} \mathrm{H}_{2} \mathrm{O}$. A 26-gauge butterfly needle was used to cannulate the trachea and bronchoalveolar lavage (BAL) was performed by instilling $0.5 \mathrm{~mL}$ phosphate-buffered saline $+5 \mathrm{mM}$ EDTA solution as previously described (27). Lungs were lavaged under constant pressure of $25 \mathrm{~cm} \mathrm{H}_{2} \mathrm{O}$ and retrieved solutions were centrifuged at $900 \times g$ for 15 minutes. The cell-free supernatants were assayed by specific ELISAs and collected cells were analyzed for total cell counts and cytospin differentials. Lung tissue was then processed for the following: calculation of lung edema; mRNA; ELISA analysis; and histopathological and morphometric analysis by fixing in $4 \%$ paraformaldehyde at $30 \mathrm{~cm} \mathrm{H}_{2} \mathrm{O}$ pressure inflation and embedded in paraffin.

Histopathologic and morphometric analysis of lungs. In lung inflammation, three random 5- $\mu$ paraffin-embedded tissue sections were taken from four different lungs of dsRNA-treated or ssRNA-treated mice at 3 days after intratracheal injection, and from age-matched 13-day-old untreated control mice. The tissue sections were stained with hematoxylin and eosin (H\&E) and lung histopathology was examined in a blinded manner as to what group/ mouse was reviewed using a modification of a histologic scoring system as previously described (28). Four easily identifiable pathologic processes were scored on a scale of 0 to 4 as previously described (28): (a) alveolar conges- 
tion; (b) hemorrhage; (c) leukocyte infiltration or aggregation of neutrophils in airspace or the vessel wall; and (d) thickness of the alveolar wall. A score of 0 represented normal lungs; 1 , mild $<25 \%$ lung involvement; 2 , moderate $25 \%$ to $50 \%$ lung involvement; 3 , severe $50 \%$ to $75 \%$ lung involvement; and 4 , very severe $>75 \%$ lung involvement. An overall score of lung injury was obtained based on the summation of all the scores and then a mean \pm SEM was generated from the cohort of ssRNA or dsRNA-treated lungs ( 3 sections from each lung, 4 lungs per group) to generate a cumulative histologic lung injury score.

For assessment of alveolization, three random $5-\mu$ paraffin-embedded tissue sections were taken from four different lungs of dsRNA-treated or ssRNAtreated mice at 5 days after intratracheal injection, and from age-matched 15-day-old untreated control mice. The tissue sections were stained with hematoxylin and eosin. and lung histopathology was reviewed in a blinded manner as to what group/mouse was reviewed using a modification of an alveolization scoring system calculating mean linear intercept as previously described (29). The number of alveolar intercepts was calculated by counting the number of times an alveolar wall intersected a line of finite length $(20 \mathrm{~mm})$ placed at random upon the lung field at $200 \times$ magnification. A lower number of alveolar intercepts reflects larger airspaces suggesting decreased alveolization. A minimum of 10 measurements were conducted per field for a total of thirty measurements per animal. Furthermore, extreme care was used to ensure equal standardization of all tissue samples by fixing at $30 \mathrm{~cm} \mathrm{H}_{2} \mathrm{O}$ inflation pressure.

Total RNA isolation and real-time quantitative TaqMan reversetranscriptase polymerase chain reaction. Total cellular RNA from lung tissue was isolated as previous described $(25,30)$. Total RNA was determined and 1 $\mu \mathrm{g}$ of total RNA was reversed-transcribed into cDNA and amplified using TaqMan Gene Expression Quantification assays (Applied Biosystems, Foster City, CA; Kit 4304134). cDNA was amplified and quantified using the TaqMan 7700 Sequence Detection System and specific primers for murine CXCL1, murine CXCL2/3, murine CXCR2, and a housekeeping gene18-s. The primers used were 5'-TGA-GCT-GCG-CTG-TCA-GTG-CCT-3' (sense) and 5'-AGA-AGC-CAG-CGT-TCA-CCA-GA-3' (antisense) for CXCL1 (259 bp) and 5'-GCT-GGC-CAC-CAA-CCA-CCA-GG-3' (sense) and 5'-AGCGAG-GCA-CAT-CAG-GTA-CG-3' (antisense) for murine CXCL2/3 (359 bp). Predeveloped assay reagents (Applied Biosystems Kit 4304134) were used for murine CXCR2 and the housekeeping gene, 18S. Quantitative analysis of gene expression was performed using the comparative $C_{\mathrm{T}}\left(\Delta C_{\mathrm{T}}\right)$ methods, in which $C_{\mathrm{T}}$ is the threshold cycle number (the minimum number of cycles needed before the product can be detected) $(22,23)$. The arithmetic formula for the $\Delta C_{\mathrm{T}}$ method is the difference in threshold cycles for a target (i.e., CXCR2) and an endogenous reference (i.e., housekeeping gene 18S). The amount of target normalized to an endogenous reference (i.e., CXCR2 in dsRNA-treated animals) and relative to a calibration normalized to an endogenous reference (i.e., CXCR2 in ssRNA-treated controls) is given by $2^{-\Delta \Delta} \mathrm{CT}$ $(22,23)$. The following is an example for comparing CXCR2 expression from dsRNA-treated animals and ssRNA-treated controls. Both CXCR2 from dsRNA-treated and ssRNA-treated controls were normalized to $18 \mathrm{~S}: \Delta \Delta C_{\mathrm{T}}=$ $\Delta C_{\mathrm{T}}$ (CXCR2 expression from dsRNA-treated animals normalized to endogenous $18 \mathrm{~S})-\Delta C_{\mathrm{T}}$ (CXCR2 expression from ssRNA-treated controls normalized to endogenous $18 \mathrm{~S}$ ). The calculation of $2^{-\Delta \Delta} \mathrm{CT}$ then gives a relative value when comparing the target with the calibrator, which we designate in this context as fold increase of dsRNA-treated animals to ssRNA-treated controls of the target mRNA relative quantification.

Lung edema analysis related to lung injury. To quantitate lung edema following dsRNA treatment, wet-to-dry weight ratios were obtained by ligating the lungs away from the hilum as previously described (23). The lungs were blotted dry and weighed. They were then desiccated by incubation at $130^{\circ} \mathrm{C}$ overnight in a vacuum oven and re-weighed to determine their dry weight. The wet to dry ratio was then calculated.

CXCL1 and CXCL2/3 ELISAs. Murine CXCL1 or CXCL2/3 protein was quantitated using a modification of a double ligand method as previously described $(23,25)$. Briefly, flat-bottomed 96-well microtiter plates (Nunc Immuno-Plate I 96-F) were coated with anti-murine CXCL1 or anti-murine CXCL2/3 $\left(1 \mathrm{ng} / \mu \mathrm{L}\right.$ in $0.6 \mathrm{M} \mathrm{NaCl}, 0.26 \mathrm{M} \mathrm{H}_{3} \mathrm{BO}_{4}$, and $\left.0.08 \mathrm{~N} \mathrm{NaOH}, \mathrm{pH} 9.6\right)$ for 24 hours at $4^{\circ} \mathrm{C}$ and then washed with phosphate-buffered saline, $\mathrm{pH} 7.5$, $0.05 \%$ Tween-20 (wash buffer). Microtiter plate nonspecific binding sites were blocked with $2 \%$ bovine serum albumin in phosphate-buffered saline and incubated for 60 minutes at $37^{\circ} \mathrm{C}$. Plates were washed three times with wash buffer and samples or standard were added, followed by incubation for 1 hour at $37^{\circ} \mathrm{C}$. Plates were washed three times and $50 \mu \mathrm{L} /$ well of biotinylated anti-murine CXCL1 antibody or anti-murine CXCL2/3 added, and plates were incubated for 45 minutes at $37^{\circ} \mathrm{C}$. Plates were washed three times, streptavidinperoxidase conjugate (Jackson Laboratories, West Grove, PA) added, and the plates incubated for 30 minutes at $37^{\circ} \mathrm{C}$. Plates were washed three times and TMB (3,3,'5,5'-tetramethylbenzidine) chromogen substrate (Kirkegaard \&
Perry Laboratories, Gaithersburg, MD) added. The plates were incubated at room temperature to the desired extinction, and the reaction terminated with 3 $\mathrm{M} \mathrm{H}_{2} \mathrm{SO}_{4}$ solution. Plates were read at $450 \mathrm{~nm}$ in an automated microplate reader (Bio-Tek Instruments, Inc., Winooski, VT). Standards were $1 / 2-\log$ dilutions of either CXCL1 or CXCL2/3 from $100 \mathrm{ng}$ to $1 \mathrm{pg} / \mathrm{mL}(50 \mu \mathrm{L} / \mathrm{well})$. This ELISA method consistently detected specific chemokine concentrations in a linear fashion more than $50 \mathrm{pg} / \mathrm{mL}$. CXCL1 and CXCL2/3 were specific in our sandwich ELISA without cross-reactivity to a panel of cytokines including human and murine IL-1Ra, IL-1, IL-2, IL-6, IL-4, tumor necrosis factor- $\alpha$, interferon- $\gamma$, and members of the CXC and CC chemokine families.

FACS analysis of lung neutrophils. . Whole-lung single-cell suspensions were made from harvested lungs from four mice per group using a method as previously described (23). Single-cell suspensions $\left(5 \times 10^{6}\right.$ cells $\left./ \mathrm{mL}\right)$ were stained with Abs: Tricolor-conjugated (BD Biosciences, Franklin Lakes, NJ) anti-murine CD45 (Caltag Laboratories, South San Francisco, CA), RPhycoerythrin (R-PE) conjugated rat anti-murine Ly-6G (neutrophil surface marker; BD Biosciences). Dual-color-stained cell suspensions were analyzed on a FACScan flow cytometer (Becton Dickinson Immunocytometry Systems, San Jose, CA) using CellQuest 3.2.1f1 software (BD Immunocytometry Systems).

Statistical analysis. . Data were analyzed using the Microsoft@ Excel 2000 statistical package (Microsoft Corporation). Two group comparisons were evaluated using the unpaired $t$ test. Three group comparisons were evaluated by the ANOVA test with the post hoc analysis (i.e., Bonferroni/Dunn). Data were expressed as mean $\pm \mathrm{SEM}$.

\section{RESULTS}

DsRNA decreases alveolization. Data on the role of inflammation resulting in decreased alveolization is currently limited to rodent models of hyperoxia-induced lung injury. We set out to determine whether dsRNA-induced inflammation could lead to decreased alveolization in a newborn mouse model undergoing ongoing lung development. Ten-day-old BALB/c mice treated with intratracheal dsRNA were noted to have significantly decreased alveolization at 5 days after treatment compared with ssRNA-treated and age-matched untreated controls. Decreased alveolization was reflected by an increased size of airspaces and fewer number of alveolar wall intercepts (Fig. 1A and $B$ ). Importantly, we exercised extreme care in ensuring that all samples were equally inflated and fixed on the same apparatus with $4 \%$ paraformaldehyde at $30 \mathrm{~cm} \mathrm{H}_{2} \mathrm{O}$ inflation pressure.

DsRNA induces lung inflammation. With the finding that dsRNA decreased alveolization as early as 5 days after intratracheal exposure, we next focused on the elicited inflammatory response to begin to dissect the mechanism(s) related to this process. Ten-day-old BALB/c mice were noted to have maximal intraparenchymal and airway leukocyte infiltration at 72 hours after intratracheal dsRNA treatment compared with ssRNA-treated and age-matched untreated controls as demonstrated by histopathology (Fig. 2A). No significant differences were noted among the histopathologic groups at earlier time points of 24 and 48 hours after dsRNA treatment (data not shown). Interestingly, lung histopathology at 5 days after dsRNA treatment showed resolution of leukocytic infiltrate while maintaining significant differences in alveolization as shown previously in Figure $1 A$. We thus chose this observation as the basis to focus on the 72-hour time point wherein the potential role of inflammation on alveolization might be greatest. FACS (fluorescence-activated cell sorting) and BAL analysis at 72 hours after dsRNA treatment specifically showed that dsRNA induced significant neutrophil recruitment compared with controls (Fig. $2 B$ and $C$ ). 

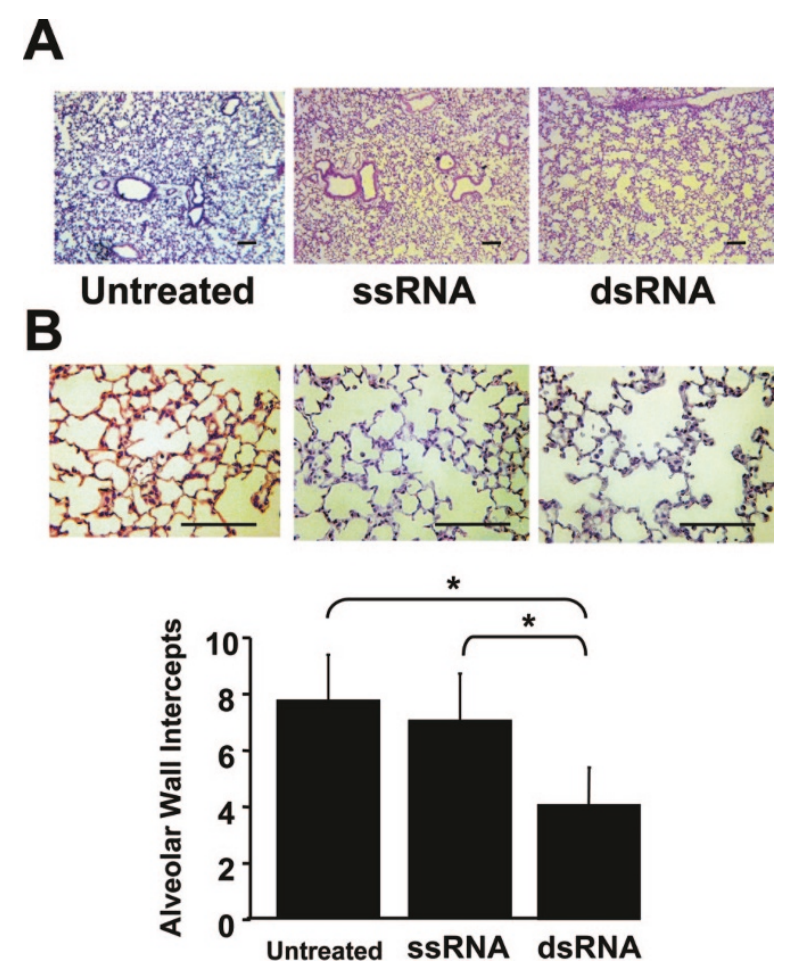

Figure 1. dsRNA decreases alveolization. (A) Histopathology of lung at 5 days after intratracheal treatment with ssRNA or dsRNA and age-matched 15-day-old untreated controls. Representative photomicrographs with H \& E staining ( $40 \times ; n=4$ mice per group). (B) Magnified representative photomicrographs $(200 \times)$ as in $(A)$ with standard line of finite length used to calculate number of alveolar wall intercepts (bottom panel, $n=4$ mice per group, 3 slides per mouse, and 10 random measurements per slide; $* p<0.05$ ). Scale bar is $100 \mu \mathrm{m}$.

DsRNA induces lung injury. To determine whether the influx of neutrophils into lung airways and parenchyma results in lung injury, we measured a lung injury score and measured lung wet-to-dry ratio to quantify changes in lung edema. Quantitative analysis of lung histopathology showed significant increases in leukocytic infiltrate, alveolar thickness, and cumulative inflammatory score at 72 hours after dsRNA treatment compared with ssRNA-treated controls (Fig. 3A). Similarly, results showed a significant increase in the wet-to-dry ratio in dsRNA-treated lungs compared with controls (Fig. 3B).

DsRNA induces elevated CXCL1 mRNA and protein levels. Because neutrophil influx was associated with lung injury in dsRNA-treated lungs, we next identified which specific factors, such as chemokines, were responsible for neutrophil recruitment. We focused on the ELR-positive CXC chemokines, CXCL1 and CXCL2/3, that are known to have neutrophil chemoattractant properties. DsRNA treatment resulted in a significant increase in lung CXCL1 mRNA levels as well as in protein levels from whole lung tissue homogenates and BAL when compared with controls at 72 hours after dsRNA treatment (Fig. $4 A$ and $B$ ). However, in contrast to levels of CXCL1, we did not find elevated levels of CXCL2/3 at either mRNA or protein levels (Fig. $4 A$ and $B$ ).

DsRNA increases CXCR2 expression in lungs. CXCR2 is the cellular receptor for the murine ELR-positive CXC chemokines CXCL1 and CXCL2/3 (18). The finding of increased
A
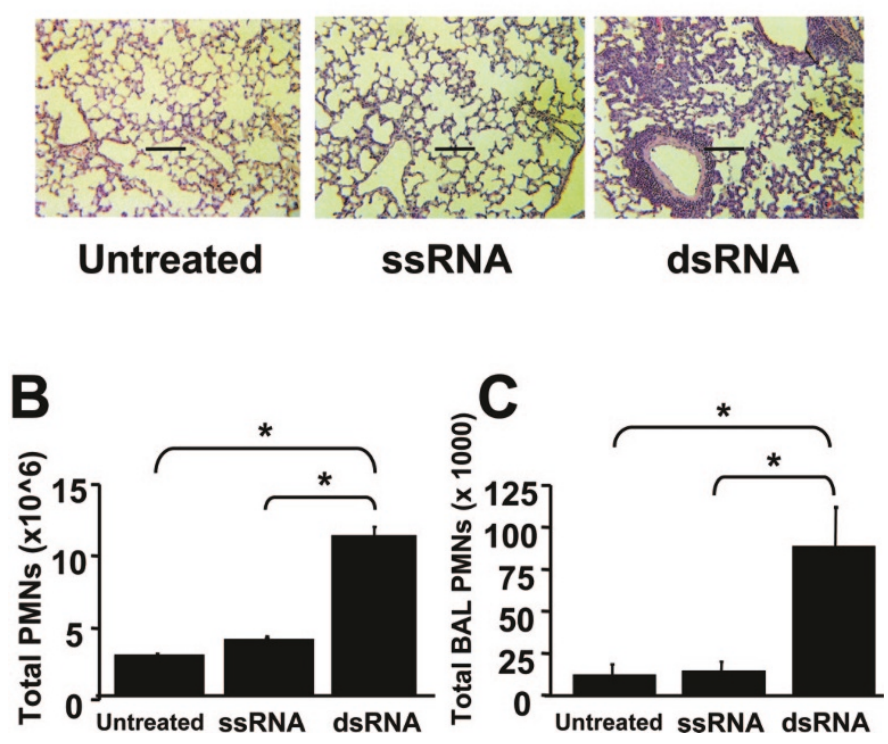

Figure 2. dsRNA induces lung neutrophil infiltration. (A) Histopathology of lung at 72 hours after treatment with ssRNA or dsRNA and age-matched 13-day-old untreated controls. Representative photomicrographs with $\mathrm{H} \& \mathrm{E}$ staining $(100 \times ; n=4$ mice per group). (B) Total lung PMNs via FACS analysis of whole-lung single cell suspensions at 72 hours $(n=4$ mice per group; $* p<0.05)$. (C) Total PMNs via BAL fluid cell count at 72 hours $(n=$ 4 mice per group; $\left.{ }^{*} p<0.05\right)$. Scale bar is $100 \mu \mathrm{m}$.

levels of CXCL1 associated with dsRNA-induced neutrophil sequestration and lung injury led us to evaluate the expression of CXCR2 mRNA in the lungs of these animals. Lung tissue from dsRNA-treated animals had significantly increased CXCR2 mRNA expression compared with controls (Fig. 5). The expression of CXCR2 mRNA paralleled its ligand expression, neutrophil sequestration, and lung injury at 72 hours after dsRNA treatment (Figs. 2-4).

Inhibition of CXCR2 inhibits infiltration of neutrophils and attenuates dsRNA-induced lung injury. To betterunderstand the mechanism partly responsible for dsRNAinduced lung neutrophilia and injury, we determined whether inhibiting CXCR2 ligand interaction with CXCR2 significantly decreased neutrophil recruitment during the pathogenesis of dsRNA-induced lung injury. Ten-day-old mice were passively immunized with specific neutralizing anti-murine CXCR2 or control antibody at 24 hours, as well as 0,24 , and 48 hours after dsRNA treatment. Lungs were harvested at 72 hours and results showed that histopathologic comparison of lung fields from anti-CXCR2-pretreated mice showed marked reduction in leukocytic infiltrate compared with control animals that received normal goat serum (Fig. 6A). BAL neutrophil counts from animals that received anti-CXCR2 Ab were also significantly reduced compared with control animals that received normal goat serum (Fig. 6B). Furthermore, the lung injury score and measurement of lung edema also showed a significant decrease in alveolar congestion, leukocytic infiltrate, alveolar thickening, cumulative lung injury score, and wet-to-dry ratios in mice treated with anti-CXCR2 compared with control antibodies (Fig. 7A and $B$ ). 


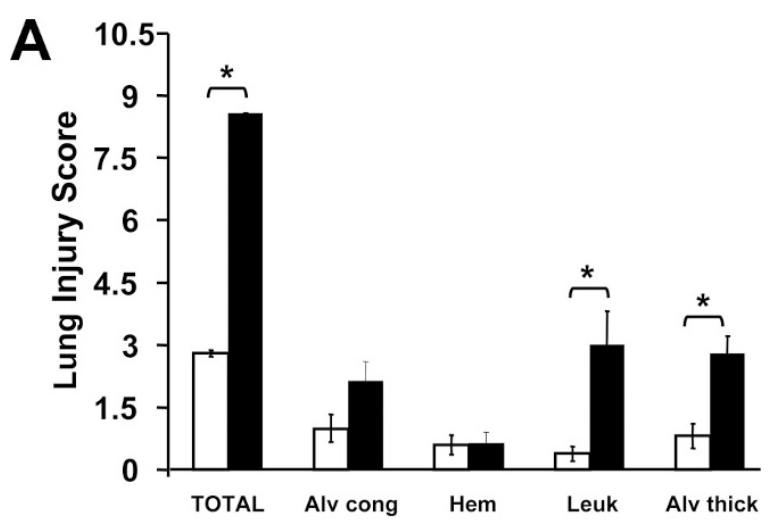

B

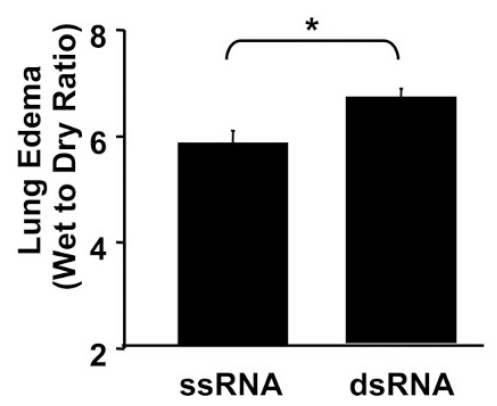

Figure 3. dsRNA induces lung injury. (A) Lung injury score calculation at 72 hours after treatment with ssRNA $(\square)$ or dsRNA ( $\square$ ). Total $=$ total cumulative score, Alv cong $=$ alveolar congestion, Hem $=$ hemorrhage, Leuk $=$ leukocytic infiltrate, Alv thick $=$ alveolar thickening $(n=4$ mice per group, 3 slides per mouse; $\left.{ }^{*} p<0.05\right)$. (B) Wet-to-dry ratio at 72 hours $(n=6$ mice per group; $* p<0.05)$.

Inhibition of CXCR2 rescues alveolization in dsRNAtreated lungs. Finally, to determine whether neutralization of CXCR2 and resultant attenuation of inflammation can rescue normal alveolization in dsRNA-treated lungs, we again compared histologic sections at 5 days after dsRNA treatment in mice pretreated with anti-murine CXCR2 or control antibodies. Results showed a preservation of alveolization as reflected by smaller airspace size and greater number of alveolar wall intercepts in anti-CXCR2 treated mice, compared with control antibody treated groups (Fig. $8 A$ and $B$ ).

\section{DISCUSSION}

The approach to dissecting the mechanisms responsible for BPD has broadly focused on three pulmonary systems including cellular responses to injury, alveologenesis, and vasculogenesis (31). Cellular responses to acute lung injury feature the neutrophil as part of innate immunity, and neutrophil infiltration has been associated with establishing a pro-inflammatory environment that may underlie the pathogenesis of BPD $(32,33)$. Potential triggers of early neutrophil influx into premature lungs include viral infections that may then injure or interrupt normal pathways during lung development and alveologenesis. The inflammation and injury during respiratory viral infections occurs because of the host's inflammatory response rather than because of the viral particles themselves (13). Viral infections are mediated by dsRNA, a proinflammatory molecule generated during viral replication. dsRNA binds
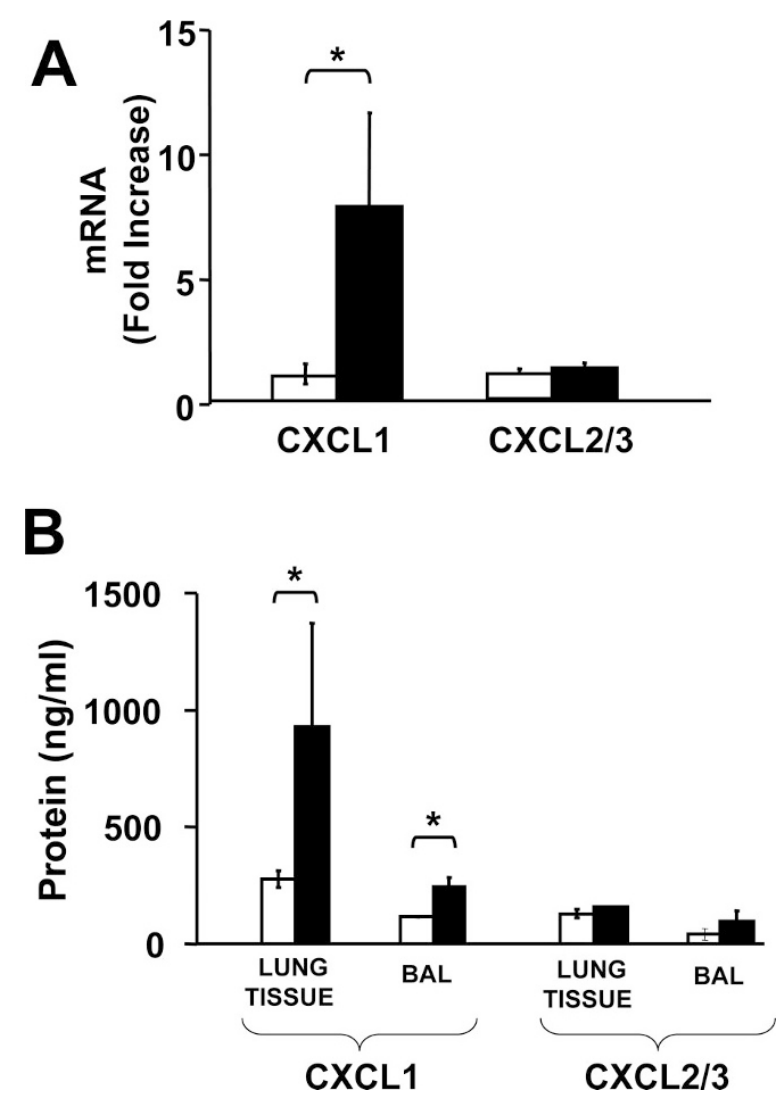

Figure 4. dsRNA induces elevated CXCL1 mRNA and protein levels. $(A)$ Quantitative levels of CXCL1 and CXCL2/3 mRNA in the lungs at 72 hours after treatment with ssRNA $(\square)$ or dsRNA $(\square)(n=6$ mice per group; $* p<$ 0.05). (B) Quantitative levels of CXCL1 and CXCL2/3 protein in the lung and BAL fluid at 72 hours $\left(n=6\right.$ mice per group; $\left.{ }^{*} p<0.05\right)$.

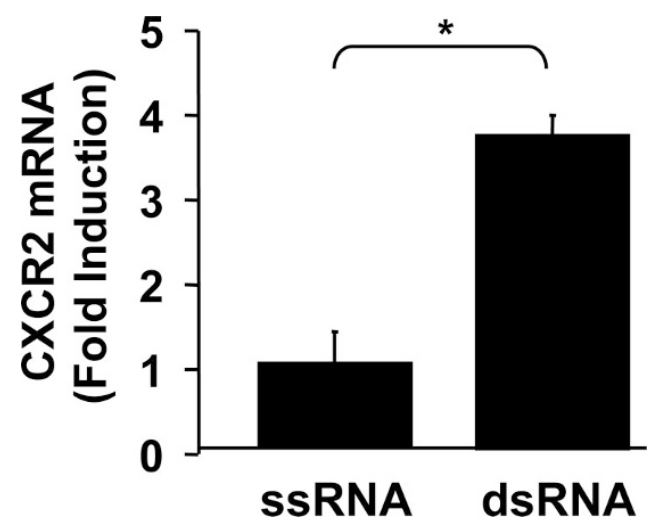

Figure 5. dsRNA increases CXCR2 expression in lungs. Quantitative realtime polymerase chain reaction was determined by TaqMan analysis for CXCR2 mRNA from lung at 72 hours after ssRNA or dsRNA treatment $(n=$ 6 mice per group; $* p<0.05)$.

to its cell surface receptor, TLR3, and activates the production of downstream gene products, such as CXC chemokines. In the current study, we hypothesized that the interaction between CXCR2 and ELR-positive CXC chemokines expressed during dsRNA-induced lung inflammation in a newborn mouse model is critical in mediating neutrophil recruitment and subsequent arrest of alveolization, a pivotal process during the pathogenesis of BPD. 


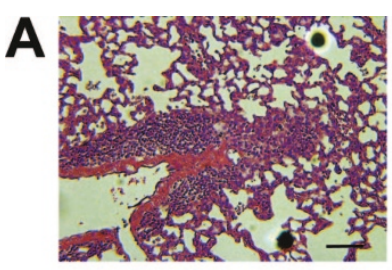

Control Ab

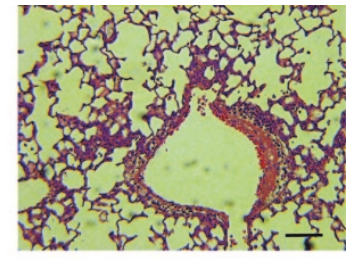

Anti-CXCR2 Ab
B

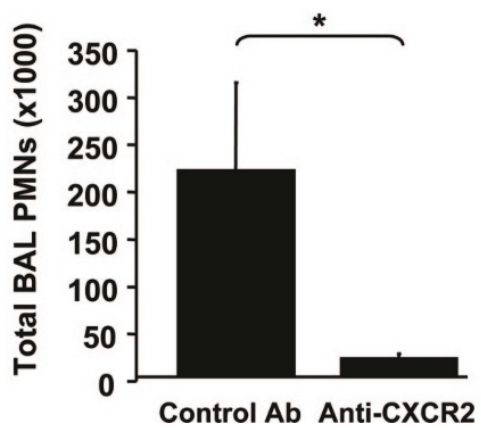

Figure 6. Inhibition of CXCR2 inhibits infiltration of neutrophils. (A) Histopathology of lungs at 72 hours after dsRNA treatment. Representative photomicrographs with $\mathrm{H} \& \mathrm{E}$ staining ( $100 \times ; n=4$ mice per group.) (B) Total PMNs via BAL fluid cell count at 72 hours $(n=4$ mice per group; ${ }^{*} p<0.05$ ). Mice were pretreated with either normal goat serum (control Ab) or anti-CXCR2 $\mathrm{Ab}$ at times $-24,0,24$, and 48 hours after IT dsRNA treatment. Scale bar is $100 \mu \mathrm{m}$.

Previous studies demonstrating decreased alveolization in rodent models have largely focused on effects of hyperoxiainduced injury, inhibition of angiogenesis or experiments utilizing transgenic animals that yield a phenotype that resembles BPD $(34,35)$. Whereas there is a growing body of literature describing decreased alveolization in transgenic mice, to our knowledge, current experimental models in wild-type animals are limited to hyperoxia exposure alone (35). Furthermore, the potential role of chemokines in decreasing alveolization has only been described in hyperoxia models and no other models have been developed to investigate the role of chemokines in mediating lung development during inflammation caused by other pathogens such as dsRNA. The present study fills this void by first demonstrating the effects of high-dose intratracheal dsRNA on neutrophil recruitment, lung injury, and decreased alveolization, and then subsequently blocking these effects and preserving normal alveolization via inhibition of the CXC chemokine receptor, CXCR2.

To determine the effects of dsRNA in newborn mouse lungs, we first characterized our newborn murine model by performing a time course of intratracheal dsRNA exposure $(50 \mu \mathrm{g} / \mathrm{g})$ to observe histopathologic changes at $0,24,48,72$, and 120 hours (5 days). Morphometric analysis demonstrated a significant decrease in alveolization in dsRNA treated versus. ssRNAtreated or age-matched untreated controls at 5 days after exposure based on comparisons of airspace size and number of alveolar wall intercepts. A maximal inflammatory response was noted at 72 hours after dsRNA exposure as reflected by significantly increased leukocytic infiltrate. We thus chose this

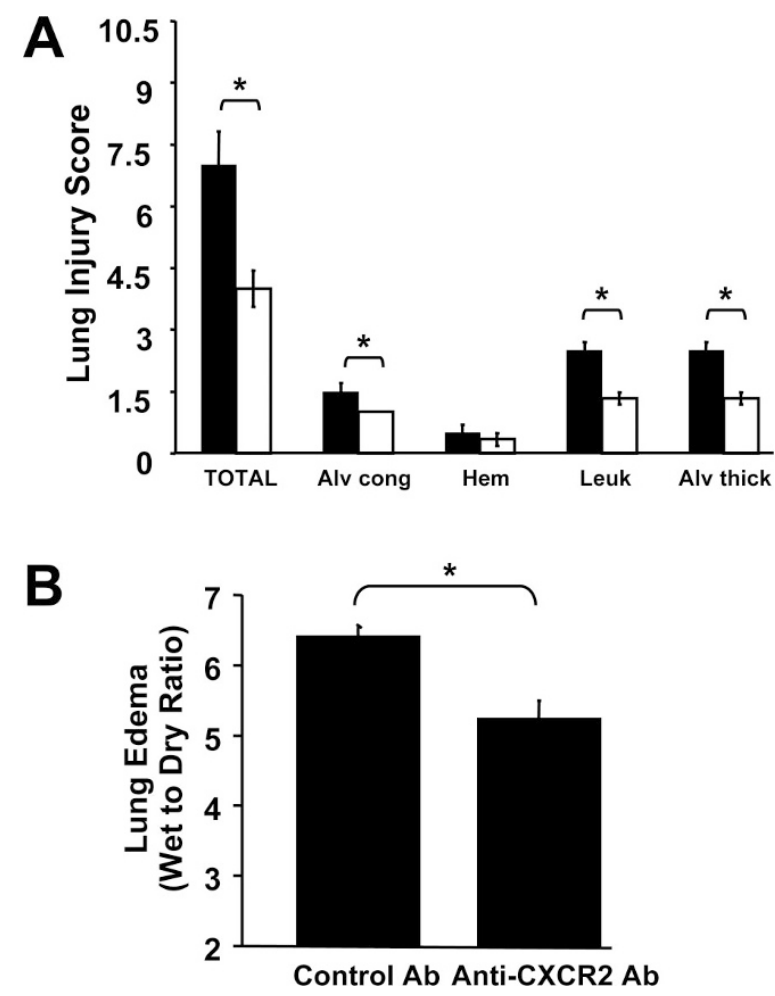

Figure 7. Inhibition of CXCR2 attenuates dsRNA-induced lung injury. $(A)$ Lung injury score calculation at 72 hours after treatment with dsRNA. Total $=$ total cumulative score, Alv cong = alveolar congestion, Hem = hemorrhage, Leuk $=$ leukocytic infiltrate, Alv thick $=$ alveolar thickening $(n=4$ mice per group, 3 slides per mouse; $* p<0.05)$. (B) Wet-to-dry ratio at 72 hours $(n=$ 6 mice per group; $\left.{ }^{*} p<0.05\right)$. Mice were pretreated with either normal goat serum (control Ab, $\square$ ) or anti-CXCR2 ( $\square$ ) Ab at times -24, 0, 24, and 48 hours after IT dsRNA treatment.

observation as the basis to focus on the 72-hour time point to dissect the inflammatory response and determine its potential role in decreasing alveolization. We also chose to use ssRNAtreated mice as controls in subsequent experiments because histology and neutrophil counts were comparable to untreated controls. Further characterization at 72 hours after intratracheal dsRNA showed that neutrophils were a predominant cell type and that there was an associated injury to alveolar-capillary membrane integrity as shown by increased alveolar thickening and lung edema.

Having characterized the histologic damage and alveolar changes caused by intratracheal dsRNA, we then focused on the underlying mechanisms responsible for promoting inflammation and injury that preceded a decrease in alveolization. Our findings of a significant increase in neutrophil infiltration following dsRNA are consistent with studies in vivo using live viruses that also resulted in early neutrophil infiltration and demonstrated that $\mathrm{CXC}$ chemokines are generated during lung inflammation (36). Because lung injury caused by dsRNA is associated with neutrophil infiltration, we determined that CXCL1 expression was significantly greater in the lungs of dsRNA-treated mice than in lungs of ssRNA-treated controls. Furthermore, expression of CXCR2 mRNA was similarly increased in parallel to the production of CXCL1 and neutrophil sequestration during dsRNA-induced lung inflammation. Whereas we have previously described the expression of 


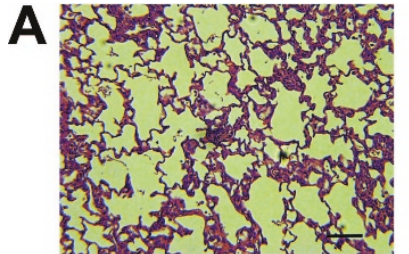

Control Ab

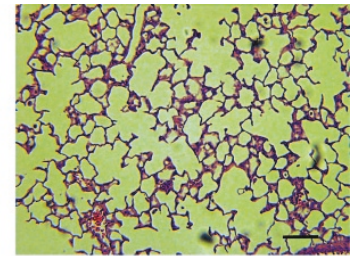

Anti-CXCR2 Ab mediating neutrophil recruitment, lung injury, and alveolization after dsRNA exposure. This mechanism may be critical in promoting a lung phenotype that resembles BPD. The findings of the current study support our contention that CXCR2/ CXCR2 ligand biology inhibits alveolization during dsRNAinduced inflammation, and may be a therapeutic target to attenuate this pathology.

\section{REFERENCES}

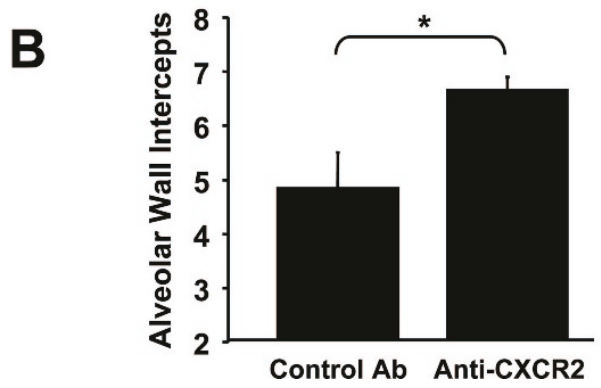

Figure 8. Inhibition of CXCR2 rescues alveolization in dsRNA-treated lungs. (A) Histopathology of lung at 5 days after intratracheal treatment with dsRNA. Representative photomicrographs with H \& E staining $(100 \times ; n=4$ mice per group). (B) Number of alveolar wall intercepts $(n=4$ mice per group, 3 slides per mouse, and 5 random measurements per slide; $* p<0.05)$. Mice were pretreated with either normal goat serum (control $\mathrm{Ab}$ ) or anti-CXCR2 Ab at times $-24,0,24,48,72$, and 96 hours after IT dsRNA treatment. Scale bar is $100 \mu \mathrm{m}$.

CXCL2/3 in the context of hyperoxia and ventilator-induced lung injury $(22,23)$, we did not find markedly elevated levels of this ELR-positive CXC chemokine in the lungs of newborn mice exposed to dsRNA. These findings suggest that dsRNA signaling may lead to differences in the expression of CXCL1 and CXCL2/3 in our model. Future studies will be designed to address this issue. Nevertheless, these results support the notion that CXCR2-dependent biology is pivotal in the recruitment of neutrophils to the lung during dsRNA-induced lung injury that contributes to decreased alveolization.

Based on these findings, we performed a proof of principle study using an antibody-mediated neutralization strategy to evaluate the direct role of CXCR2/CXCR2 ligand biology in promoting the pathogenesis of dsRNA-induced lung injury and decreased alveolization. The anti-CXCR2 Ab-treated mice demonstrated significant reductions in neutrophil infiltration that were paralleled by a decrease in lung injury at 72 hours and by the preservation of normal alveolization at 5 days after dsRNA exposure. Our study is the first to our knowledge to demonstrate that neutralization of CXCR2 results in decreased neutrophil recruitment, decreased lung injury, and preservation of normal alveolization after dsRNA exposure. Although the precise signaling mechanisms underlying the interaction between chemokines and lung developmental pathways remains to be studied, it is intriguing to consider that CXCR2 and its ligands may in fact play an important developmental role in modulating lung architecture similar to their known role in modulating angiogenesis $(37,38)$.

In conclusion, we have demonstrated that the biologic axis of CXCR2/CXCR2 ligand signaling plays a pivotal role in
1. Jobe AH, Bancalari E 2001 Bronchopulmonary dysplasia. Am J Respir Crit Care Med

2. Watterberg KL, Demers LM, Scott SM, Murphy S 1996 Chorioamnionitis and early lung inflammation in infants in whom bronchopulmonary dysplasia develops. Pediatrics 97:210-215

3. Speer CP 1999 Inflammatory mechanisms in neonatal chronic lung disease. Eur J Pediatr 158 Suppl 1:S18-S22

4. Northway WH Jr., Rosan RC, Porter DY 1967 Pulmonary disease following respirator therapy of hyaline-membrane disease. Bronchopulmonary dysplasia. N Engl J Med 276:357-368

5. Bagchi A, Viscardi RM, Taciak V, Ensor JE, McCrea KA, Hasday JD 1994 Increased premature infants is associated with the development of bronchopulmonary dysplasia. Pediatr Res 36:244-252

6. Abele-Horn M, Genzel-Boroviczeny O, Uhlig T, Zimmermann A, Peters J, Scholz M 1998 Ureaplasma urealyticum colonization and bronchopulmonary dysplasia: a comparative prospective multicentre study. Eur J Pediatr 157:1004-1011

7. De Dooy JJ, Mahieu LM, Van Bever HP 2001 The role of inflammation in the development of chronic lung disease in neonates. Eur J Pediatr 160:457-463

8. Ballard RA, Drew WL, Hufnagle KG, Riedel PA 1979 Acquired cytomegalovirus infection in preterm infants. Am J Dis Child 133:482-485

9. Couroucli XI, Welty SE, Ramsay PL, Wearden ME, Fuentes-Garcia FJ, Ni J, Jacobs TN, Towbin JA, Bowles NE 2000 Detection of microorganisms in the tracheal aspirates of preterm infants by polymerase chain reaction: association of adenovirus infection with bronchopulmonary dysplasia. Pediatr Res 47:225-232

10. Hogg JC 2000 The adenovirus and bronchopulmonary dysplasia: an association that could be causal or coincidental. Pediatr Res 47:175-176

11. Sawyer MH, Edwards DK, Spector SA 1987 Cytomegalovirus infection and bronchopulmonary dysplasia in premature infants. Am J Dis Child 141:303-305

12. Wenstrom KD, Andrews WW, Bowles NE, Towbin JA, Hauth JC, Goldenberg RL 1998 Intrauterine viral infection at the time of second trimester genetic amniocentesis. Obstet Gynecol 92:420-424

13. Subauste MC, Jacoby DB, Richards SM, Proud D 1995 Infection of a human respiratory epithelial cell line with rhinovirus. Induction of cytokine release and modulation of susceptibility to infection by cytokine exposure. J Clin Invest 96:549557

14. Gern JE, Vrtis R, Grindle KA, Swenson C, Busse WW 2000 Relationship of upper and lower airway cytokines to outcome of experimental rhinovirus infection. Am J Respir Crit Care Med 162:2226-2231

15. Gern JE, French DA, Grindle KA, Brockman-Schneider RA, Konno SI, Busse WW 2003 Double-stranded RNA induces the synthesis of specific chemokines by bronchial epithelial cells. Am J Respir Cell Mol Biol 28:731-737

16. Ghezzi F, Gomez R, Romero R, Yoon BH, Edwin SS, David C, Janisse J, Mazor M 1998 Elevated interleukin-8 concentrations in amniotic fluid of mothers whose neonates subsequently develop bronchopulmonary dysplasia. Eur J Obstet Gynecol Reprod Biol 78:5-10

17. Munshi UK, Niu JO, Siddiq MM, Parton LA 1997 Elevation of interleukin-8 and interleukin-6 precedes the influx of neutrophils in tracheal aspirates from preterm infants who develop bronchopulmonary dysplasia. Pediatr Pulmonol 24:331-336

18. Bozic CR, Gerard NP, von Uexkull-Guldenband C, Kolakowski LF Jr., Conklyn MJ, Breslow R, Showell HJ, Gerard C 1994 The murine interleukin 8 type B receptor homologue and its ligands. Expression and biological characterization. J Biol Chem 269:29355-29358

19. Jones CA, Cayabyab RG, Kwong KY, Stotts C, Wong B, Hamdan H, Minoo P, deLemos RA 1996 Undetectable interleukin (IL)-10 and persistent IL-8 expression early in hyaline membrane disease: a possible developmental basis for the predisposition to chronic lung inflammation in preterm newborns. Pediatr Res 39:966-975

20. Massaro GD, Massaro D 1996 Formation of pulmonary alveoli and gas-exchange surface area: quantitation and regulation. Annu Rev Physiol 58:73-92

21. Mehrad B, Strieter RM, Moore TA, Tsai WC, Lira SA, Standiford TJ 1999 CXC chemokine receptor-2 ligands are necessary components of neutrophil-mediated host defense in invasive pulmonary aspergillosis. J Immunol 163:6086-6094

22. Sue RD, Belperio JA, Burdick MD, Murray LA, Xue YY, Dy MC, Kwon JJ, Keane MP, Strieter RM 2004 CXCR2 is critical to hyperoxia-induced lung injury. J Immunol 172:3860-3868

23. Belperio JA, Keane MP, Burdick MD, Londhe V, Xue YY, Li K, Phillips RJ, Strieter RM 2002 Critical role for CXCR2 and CXCR2 ligands during the pathogenesis of ventilator-induced lung injury. J Clin Invest 110:1703-1716

24. Belperio JA, Dy M, Burdick MD, Xue YY, Li K, Elias JA, Keane MP 2002 Interaction of IL-13 and $\mathrm{C} 10$ in the pathogenesis of bleomycin-induced pulmonary fibrosis. Am J Respir Cell Mol Biol 27:419-427 163:1723-1729 activity of interleukin- 6 but not tumor necrosis factor-alpha in lung lavage of 
25. Keane MP, Belperio JA, Moore TA, Moore BB, Arenberg DA, Smith RE, Burdick MD, Kunkel SL, Strieter RM 1999 Neutralization of the CXC chemokine, macrophage inflammatory protein-2, attenuates bleomycin-induced pulmonary fibrosis J Immunol 162:5511-5518

26. Garcia-Ramallo E, Marques T, Prats N, Beleta J, Kunkel SL, Godessart N 2002 Resident cell chemokine expression serves as the major mechanism for leukocyte recruitment during local inflammation. J Immunol 169:6467-6473

27. Laichalk LL, Kunkel SL, Strieter RM, Danforth JM, Bailie MB, Standiford TJ 1996 Tumor necrosis factor mediates lung antibacterial host defense in murine Klebsiella pneumonia. Infect Immun 64:5211-5218

28. Imanaka H, Shimaoka M, Matsuura N, Nishimura M, Ohta N, Kiyono H 2001 Ventilator-induced lung injury is associated with neutrophil infiltration, macrophage activation, and TGF-beta 1 mRNA upregulation in rat lungs. Anesth Analg 92:428 436

29. Husain AN, Siddiqui NH, Stocker JT 1998 Pathology of arrested acinar development in postsurfactant bronchopulmonary dysplasia. Hum Pathol 29:710-717

30. Belperio JA, Burdick MD, Keane MP, Xue YY, Lynch JP, 3rd, Daugherty BL, Kunkel SL, Strieter RM 2000 The role of the CC chemokine, RANTES, in acute lung allograft rejection. J Immunol 165:461-472

31. Vicencio AG, Lee CG, Cho SJ, Eickelberg O, Chuu Y, Haddad GG, Elias JA 2004 Conditional overexpression of bioactive transforming growth factor-betal in neonatal mouse lung: a new model for bronchopulmonary dysplasia? Am J Respir Cell Mol Biol 31:650-656
32. Yi M, Jankov RP, Belcastro R, Humes D, Copland I, Shek S, Sweezey NB, Post M, Albertine KH, Auten RL, Tanswell AK 2004 Opposing effects of $60 \%$ oxygen and neutrophil influx on alveologenesis in the neonatal rat. Am J Respir Crit Care Med 170:1188-1196

33. Kim BI, Lee HE, Choi CW, Jo HS, Choi EH, Koh YY, Choi JH 2004 Increase in cord blood soluble E-selectin and tracheal aspirate neutrophils at birth and the development of new bronchopulmonary dysplasia. J Perinat Med 32:282-287

34. Dauger S, Ferkdadji L, Saumon G, Vardon G, Peuchmaur M, Gaultier C, Gallego J 2003 Neonatal exposure to $65 \%$ oxygen durably impairs lung architecture and breathing pattern in adult mice. Chest 123:530-538

35. Warner BB, Stuart LA, Papes RA, Wispe JR 1998 Functional and pathological effects of prolonged hyperoxia in neonatal mice. Am J Physiol 275:L110-L117

36. Kajon AE, Gigliotti AP, Harrod KS 2003 Acute inflammatory response and remodeling of airway epithelium after subspecies B1 human adenovirus infection of the mouse lower respiratory tract. J Med Virol 71:233-244

37. Addison CL, Daniel TO, Burdick MD, Liu H, Ehlert JE, Xue YY, Buechi L, Walz A, Richmond A, Strieter RM 2000 The CXC chemokine receptor 2, CXCR2, is the putative receptor for ELR + CXC chemokine-induced angiogenic activity. J Immunol $165: 5269-5277$

38. Keane MP, Burdick MD, Xue YY, Lutz M, Belperio JA, Strieter RM2004 The chemokine receptor, CXCR2, mediates the tumorigenic effects of ELR + CXC chemokines. Chest 125:133S 